Uniciencia. Vol. 29, No. 2, pp. 62-83. Julio-Diciembre, 2015.

ISSN Electrónico: 2215-3470

URL: www.revistas.una.ac.cr/uniciencia

Email: revistauniciencia@una.cr

DOI: http://dx.doi.org/10.15359/ru.29-2.5

\title{
Las estrategias metodológicas en el aprendizaje de la biología
}

\section{A look of the contributions of the methodological strategies in learning biology}

\author{
José Pereira-Chaves \\ jose.pereira.chaves@una.cr \\ Universidad Nacional de Costa Rica. \\ Heredia, Costa Rica.
}

Recibido-Received: 8/mar/2014 / Aceptado-Accepted: 9/set/2014 / Publicado-Published: 31/jul /2015.

\begin{abstract}
Resumen
El objetivo de esta investigación fue documentar la contribución de las estrategias metodológicas empleadas por el docente en el aprendizaje de Biología. La enseñanza de la Biología, desde el punto de vista de aprendizaje obligatorio para los estudiantes de Secundaria, se ha convertido en un gran reto para los profesores de esta disciplina. De manera que las acciones que se llevan a cabo en el aula para mediar el contenido, deben ser planificadas con una visión amplia de lo que se quiere lograr con el aprendizaje. Por ello, la importancia de entender los procesos de enseñanza y aprendizaje de los conceptos biológicos en el salón de clases es medular durante la mediación pedagógica. Esta investigación se llevó a cabo bajo un enfoque etnográfico por medio de un estudio de caso, se realizaron observaciones, entrevistas, análisis de contenido, y un grupo focal. Se evidenció la preocupación y el compromiso de la docente para que sus estudiantes comprendieran el contenido biológico mediante el uso de las diversas estrategias metodológicas, y así se interesaran para continuar aprendiendo. Por otra parte los estudiantes mostraron motivación en cada una de las actividades didácticas llevadas a cabo en el aula.
\end{abstract}

Palabras clave: estrategias; metodológica; enseñanza; aprendizaje, estudiantes; docente; interacción.

\begin{abstract}
Teaching Biology, from the point of view of mandatory learning for high school students, has become a major challenge for teachers of this discipline. Therefore, the actions carried out in the classroom to mediate the content, should be planned with a broad vision of what we want to accomplish with learning. Due to this, the importance of understanding the processes of teaching and learning of biological concepts in the classroom is crucial for pedagogical mediation. The aim of this study was to document the contributions of the methodological strategies used by the teacher in the learning of Biology. This research was carried out under an ethnographic approach through a case study, observations, interviews, content analysis, and a focus group. It was evident the concern and commitment of the teacher in the understanding of the biological content, using various methodological strategies by the students, and the encouragement given to them to continue learning. On the other hand, the students showed motivation in each of the academic activities performed in the classroom.
\end{abstract}

Key words: teaching strategies; teaching; learning; students; teacher; interaction. 
Con base en la experiencia personal como profesor de Secundaria en los último nueve años y a partir del intercambio de experiencias con colegas profesores de Biología, he comprobado un desarrollo de los conceptos biológicos, basados en la facilitación de guías de estudio o cuestionarios, que deben ser contestados por los estudiantes con un libro de texto asignado por el profesor. Esta ha sido la forma de abordar y desarrollar la mayor cantidad de contenido del programa de Biología para el Ciclo Diversificado. Dale y Ueckert (2007), destacaron en la Conferencia sobre la Enseñanza de la Biología en los Estados Unidos, que uno de los dilemas y de los desafíos más grandes de los profesores de Biología corresponden a: ¿cómo abordar un programa tan cargado de temas y tener buenos resultados en los exámenes, y a la vez lograr que el estudiante se eduque y aprenda en este proceso?

Por otra parte, Pereira (2010) en sus investigaciones señala que los profesores de biología mostraban preocupaciones en torno a cómo hacer para que los estudiantes se motiven en el proceso de aprendizaje, y sobre todo que ellos cumplan con sus obligaciones asignadas como repasar la materia, presentar los materiales que favorezcan una mejor compresión de los conceptos entre otros. Así los profesores deben promover estrategias de aula que permitan el discernimiento mental del educando.

Por otra parte la propuesta del programa de Biología para los niveles de Décimo y Undécimo años del Ciclo Diversificado del sistema educativo costarricense está elaborada para que se desarrolle en tres lecciones por semana durante el año escolar y promueve la innovación para enseñar dicho contenido. Sin embargo he visto la resistencia o poca motivación que tienen los profesores de Biología para cambiar sus estrategias metodológicas tradicionales, como lo son las clases magistrales y el desarrollo de guías de trabajo, porque probablemente ellos están preparando al estudiante para una prueba nacional de Bachillerato y concentrando todo el aprendizaje en la definición de conceptos y aspecto que podría provocar en el alumno poca motivación y por ende agobio y poca atracción por esta área de las ciencias.

Se evidencia la poca aplicación del desarrollo conceptual y su interpretación, donde se promueva el interés de interpretar y dar sentido a todo lo que nos rodea, por eso para que se geste una verdadera educación científica es necesario provocar no sólo un abordaje adecuado de los textos que están llenos de información y poco atractivos para el estudiante, sino es que a través de ellos se comprenda de modo pertinente la realidad cotidiana y se provoque la aplicación del nuevo conocimiento para la vida.

Por eso se llama a la reflexión para que se profundice en nuestra labor docente y se autoevalúe si realmente en las clases de biología se está provocando la adquisición del conocimiento científico, teniendo en cuenta que un pedagogo no debe quedar en el intento de transmitir a sus estudiantes los conocimientos, sino a través de su mediación en el aula, provocar el interés en cada uno de ellos y sobre todo el sentido de utilidad y relación con el entorno de lo que aprende, para que no sólo le sea útil para la vida, sino que sea capaz de tomar decisiones responsables y consientes a partir de ellos. 
Para el desarrollo, mediación o promoción del conocimiento son muchas las alternativas disponibles para su adecuada aplicación. La diferencia de cómo potenciarlas la hace el docente, quien debe poner en práctica la imaginación, la creatividad y el interés, para facilitarles a sus estudiantes de la mejor manera, los conceptos que deben interiorizar. Además las técnicas didácticas, deben ser valoradas de acorde al contenido y de lo que se pretende alcanzar con el mismo, por ejemplo si se hace una presentación oral, esquemas, resúmenes, si se quiere que el estudiante repita el contenido, transforme o reconstruya la información, y a la vez se gesten actividades de apoyo constante.

Por otra parte, para abordar el contenido se requiere del apoyo de técnicas didácticas que favorezcan la mediación, el estímulo y la interiorización conceptual de los contenidos propuestos en los objetivos de aprendizaje y van de la mano con los diferentes modelos de formación que tenga el profesor y con las técnicas didácticas de enseñanza, por ejemplo, entre las más usadas están: la mesa redonda, explicación, discusión de grupo, demostraciones, investigaciones, estudios de casos, cuestionarios, resumen, comentarios de lecturas, entre otros.

Otro de los aspectos a considerar en la labor docente, es el reconocimiento de los aspectos teórico- pedagógicos, que podrían mejorar e incentivar el aprendizaje, según la tendencia o afinidad del docente, por ello se resaltan los desafíos que enfrentan en la actualidad, donde Allen (2006), reseña lo escrito por Evaney y Sykes (1988). El docente debe ayudar a los alumnos a elaborar construcciones cognitivas complejas, además que aborden problemas más estructurados, así como preparar al estudiante para que organice y supervise su propio aprendizaje, que incorpore en las estrategias metodológicas los conocimientos previos altamente específicos, donde se resalte en ellos el aprendizaje cooperativo y correspondiente a la situación, y a la vez se potencien las destrezas de cada uno de ellos, el desarrollo de tareas. (p.5).

\section{Metodología}

La investigación se desarrolló en un aula del Colegio Humanístico Costarricense de Heredia, en el país sólo hay dos centros educativos con esta modalidad y este por ser el primero y tener destacados resultados académicos en el área de biología, como en las olimpiadas costarricense de ciencias biológicas, inclusive superando centros educativos con modalidad científica, interesó documentar la actividad académica, para ello se realizó una investigación cualitativa, mediante el método etnográfico con un estudio de caso, con el único grupo de undécimo año que tenía la institución, integrado por 24 estudiantes (12 hombre y 12 mujeres), se realizaron 18 observaciones participantes(OP) los jueves de 8:00 a 10:15 am comprendido entre febrero y setiembre del 2011.

Rodríguez, Gil, y García (1996) establecen que la observación en el aula permite obtener información sobre un fenómeno o acontecimiento, tal y como este se produce. Para ello, durante la investigación se estuvo inmerso en el desarrollo de las actividades en el 
aula, de manera que se documentaron in situ, los sentimientos de gusto, disgusto, resistencia, apatía hacia los contenidos de biología que fueron evaluados.

A la vez se les aplicó una entrevista semiestructurada por duplicado que se planificó en seis partes, en total se entrevistaron a 12 estudiantes (EE) y 5 a la profesora (EP), los estudiantes fueron seleccionado según reacciones identificadas durante las observaciones, para lo cual se realizaron una serie de preguntas abiertas, previamente definidas, con un guion de entrevista que podría variar en la secuencia y formulación según características y respuestas que se iban obteniendo de cada entrevistado.

Se realizaron dos sesiones con un grupo focal con seis estudiantes, cuyo criterio de selección fueron las dos con notas más bajas y más altas y dos que estaban dentro del promedio (84), está técnica permitió la discusión y confrontación de las percepciones individuales de los estudiantes. Lo que facilitó la diferenciación de los puntos de vista colectivos e individuales.

La información fue analizada referente a las estrategias metodológicas y se fueron agrupando en subcategoría por ejemplo: Recursos didácticos(abordaje del libro de texto y revistas, uso de materiales tradicionales, caseros, recursos del entorno), recursos didácticos (uso de la pizarra, recursos audiovisuales, interacción entre docente y estudiantes-estudiante estudiante, trabajo individual, grupal), relación teoría práctica (experiencia de campo, articulación de conocimientos previos como por ejemplo a partir de lo que el estudiante sabe comenzar a desarrollar la parte conceptual), uso del lenguaje biológico(lenguaje científico, técnico y articulado).

Se realizó el análisis de validez de contenido de cada instrumento en esta investigación, empleando la técnica del juicio de expertos para identificar posibles erros de elaboración y compresión y así realizar los ajustes pertinentes antes de aplicarlos durante la investigación, para tal efecto se contó con 5 profesores universitarios de la Escuela de Ciencias Biológicas, quienes tenían grado de escolaridad de Máster con una formación de biólogos y en el componente pedagógico, a la vez se les aplicó a 5 profesores de biología en Secundaria con el grado de licenciatura.

Para verificar los ajustes se les aplicó a 10 estudiantes de la Escuela de Ciencias Biológicas de la Universidad Nacional de Tercer Año de carrera de la Enseñanza de las Ciencias Naturales.

\section{Resultados y análisis}

Para la profesora, el programa de Biología del Ciclo Diversificado es bastante completo en cuanto a contenidos, dado que da una visión amplia de los mismos, desde las sustancias del protoplasma, evolución, célula, homeostasis, etc., con lo cual, en dos años los estudiantes salen con una visión amplia de una Biología general. Sin embargo, la docente considera que el desarrollo de estos contenidos se ven afectado por el factor tiempo en el aula. Además enfatiza en que este programa evidencia el buen nivel que se busca alcanzar en materias científicas para Costa Rica. Sin embargo otro de los aspectos limitantes para el 
desarrollo de los contenidos son los recursos audiovisuales y escritos, no siempre están disponibles en todos los centros educativos, principalmente considerando que los libros de Secundaria no se ajustan a estos contenidos, según lo expresan los colegas de otras instituciones. Tal problema no se da en este centro educativo, porque aquí se dispone de todos los recursos necesarios. Por eso hacemos la diferencia con el abordaje de Biología en la institución y realmente estamos satisfechos con el nivel de logro y aplicación conceptual por parte de los estudiantes. (EP1).

Uno de los aspectos a resaltar es que la asignatura de Biología en la mayoría de las ocasiones es abordada mediante el desarrollo de conceptos generales, entre ellos: diversidad, la vida, los organismos, sin que se haga el esfuerzo hacer referencia a los seres vivos desde un punto de vista sistémico y tener en cuenta características como el holismo, la sinergia y el mantenimiento del equilibrio ambiental entre otras. En esta línea Valbuena (2008), señala que los docentes deben tener la habilidad de presentar el contenido a sus estudiantes de forma amena.

El contenido de un curso de Biología no debe ser únicamente aplicando la memoria, sino, el docente debe articular diversos recursos didácticos para la construcción y aplicación de los conocimientos teóricos, referente a los diversos y complejos procesos que conllevan la vida, a la vez promover actitudes y valores que contribuyan a mejorar, usar y conservar los recursos naturales, así como todas las implicaciones que se dan en torno a la vida y su completa interacción y comprensión.

Este conocimiento deberá ir enfocado a la resolución de problemas y a la promoción de conductas responsables, conjugando experiencias y habilidades del docente con la puesta en escena de distintos abordajes metodológicos, que promuevan en los estudiantes el desarrollo de capacidades para aplicar procedimientos, conceptos, actitudes, habilidades y vivencias, en torno a la adquisición de los conocimientos de la Biología como ciencia.

En este sentido en las entrevistas aplicadas a los estudiantes ellos señalan que la metodología que se desarrolla en clases es fundamental, señalan que un profesor de Biología debe de ponerse a prueba antes de ser contratado, porque no cualquier persona puede enseñar Biología, tiene que ser un profesor experimentado, que profundice los temas, porque si no va el estudiante a quedar un poco desmotivado, lo que es muy cierto; imagínese que toda la materia que se ve no es fácil. Aquí el proceso es muy dinámico y la profesora usa diferentes técnicas que le facilitan a uno aprender más fácilmente, con una pedagogía más aplicada, en la que no sólo es la memoria, la idea es divertirse en la Biología y amarla, y culminamos con cosas que todos esperamos en algún momento, como los laboratorios y las giras de campo, donde realmente se vive el aprendizaje e igual así se nos evalúa. (EE3).

Aquí se evidencia lo que resalta Martínez, García y Quintana (2006) quienes consideran que es fundamental que se haga una evaluación del docente, enfocada a valorar la forma de facilitar el contenido, de motivar a sus estudiantes lo cual debe trascender el espacio concreto de las aulas y de lo que se hace en ellas. 
Con base en el planteamiento anterior, es importante resaltar que la orientación pedagógica que reciben los estudiantes debe centrarse en fomentar la capacidad de aprendizaje autónomo, o sea, haciendo uso de las tendencias educativas del aprender a aprender, la cual se refiere al aprendizaje a lo largo de la vida, es decir, a las habilidades para continuar aprendiendo de manera eficaz y autónoma, una vez finalizada la etapa escolar. Esto implica, además de tener conciencia y control de las propias capacidades y conocimientos y estar debidamente motivado, el saber utilizar adecuadamente estrategias y técnicas de estudio, con el acompañamiento del docente (Acosta y Acosta, 2010).

Es necesario que se les den las herramientas a los alumnos, para que desarrollen las competencias científicas y académicas de aprendizaje que les servirán en el futuro, cuando se enfrenten a la educación superior, promover la aplicación y el dominio de herramientas de aprendizaje, que les permitan desenvolverse para la vida, lo que favorecerá personas integralmente formadas. Así la vivencia de los conceptos mediante las diversas estrategias, son ejercicios enfocados a las actividades de aprendizaje basadas en el disfrute por aprender protagónicamente por parte del estudiante.

El docente en sus estrategias metodológicas, tiene que buscar diversas opciones para que los estudiantes logren comprender el contenido, que sean capaces de reflexionar sobre ellos. Pero esto no se logra solamente con la aplicación metódica de una técnica didáctica, sino que el profesor debe exigirse a sí mismo y desarrollar o poner en prácticas habilidades metacognitivas, que promuevan una conciencia sobre la forma de llevarse a cabo los procesos cognitivos; esto, con el fin de que los contenidos sean constantemente confrontados con base en las habilidades, destrezas y resultados que se van obteniendo en el proceso. Así se reflexiona sobre la necesidad de un conocimiento útil para la vida, de lo que se aprende y cómo se adquiere, donde se incluyan procedimientos, acciones que estimulen la habilidad en los estudiantes, de manera que logren un aprendizaje, que sean capaces de aplicarlo y relacionarlo con su modo de vida en las diferentes etapas de su desarrollo, como lo es la resolución de problemas y las necesidades académicas futuras. El profesor, por medio de sus diversas técnicas didácticas y su claridad conceptual, les brindará a sus estudiantes las herramientas necesarias para la obtener la información requerida y el logro de los objetivos de aprendizaje planteados.

La docente sujeto de investigación, busca siempre en su quehacer, estimular la imaginación y creatividad de los estudiantes dentro y fuera del aula, con lo que se evidencia durante las observaciones realizadas, lo señalado por Alterio y Ruíz (2010). Estos autores señalan que las estrategias de enseñanza deben ser herramientas que permitan realizar múltiples funciones en la actividad pedagógica, como realizar diagnósticos previos, monitorear conscientemente el proceso de aprendizaje, mejorar el nivel de atención y comprensión, generar buenas expectativas, fomentar la creatividad y estimular las habilidades del pensamiento.

Díaz (2002), señala que las estrategias de enseñanza y aprendizaje, antes de considerarse antagónicas, deben considerarse complementarias en el proceso de enseñanza y aprendizaje, con la finalidad de lograr que el aprendiz sea más autónomo y reflexivo. Lo

José Pereira-Chaves

Artículo protegido por licencia Creative Commons: BY-NC-ND / Protected by Creative Commons: BY-NC-ND

Revista de acceso y publicación gratuita/ Access and publication in Uniciencia is totally no fee. 
cual está de acorde con los datos obtenidos en la investigación donde se reflejó el dominio de la docente de los diversos recursos didácticos para que sus estudiantes comprendieran de una manera más dinámica, donde los estudiantes logran realmente ser proactivos en su aprendizaje y aprecian las variadas formas en que la profesora les presenta el contenido; por ello consideran, que las clases, aparte de ser provechosas son muy interesantes.

Por tal razón se debe hacer uso de variadas fuentes de información, promover durante la acción cotidiana las estrategia metodológica que se logra realizar mediante técnicas didácticas como lluvia de ideas, foros, trabajos en grupo, etc. y aprovechar la ocasión de canalizar todo el potencial que tienen los adolescentes, para provocar su interés hacia las ciencias biológicas. Además de las estrategias metodológicas entra en escena los materiales didácticos utilizados en diversas actividades. Aquí la habilidad del docente es fundamental para aplicarlos en el desarrollo del contenido. Por ejemplo, durante la etnografía desarrollada lo que se puede apreciar en la siguiente triangulación, donde se evidencia el uso de las tecnologías de la información y comunicación (TICs) en la clase como recursos didácticas que se registraron en los distintos momentos de recolección de datos, los cuales se muestran en la figura 1.

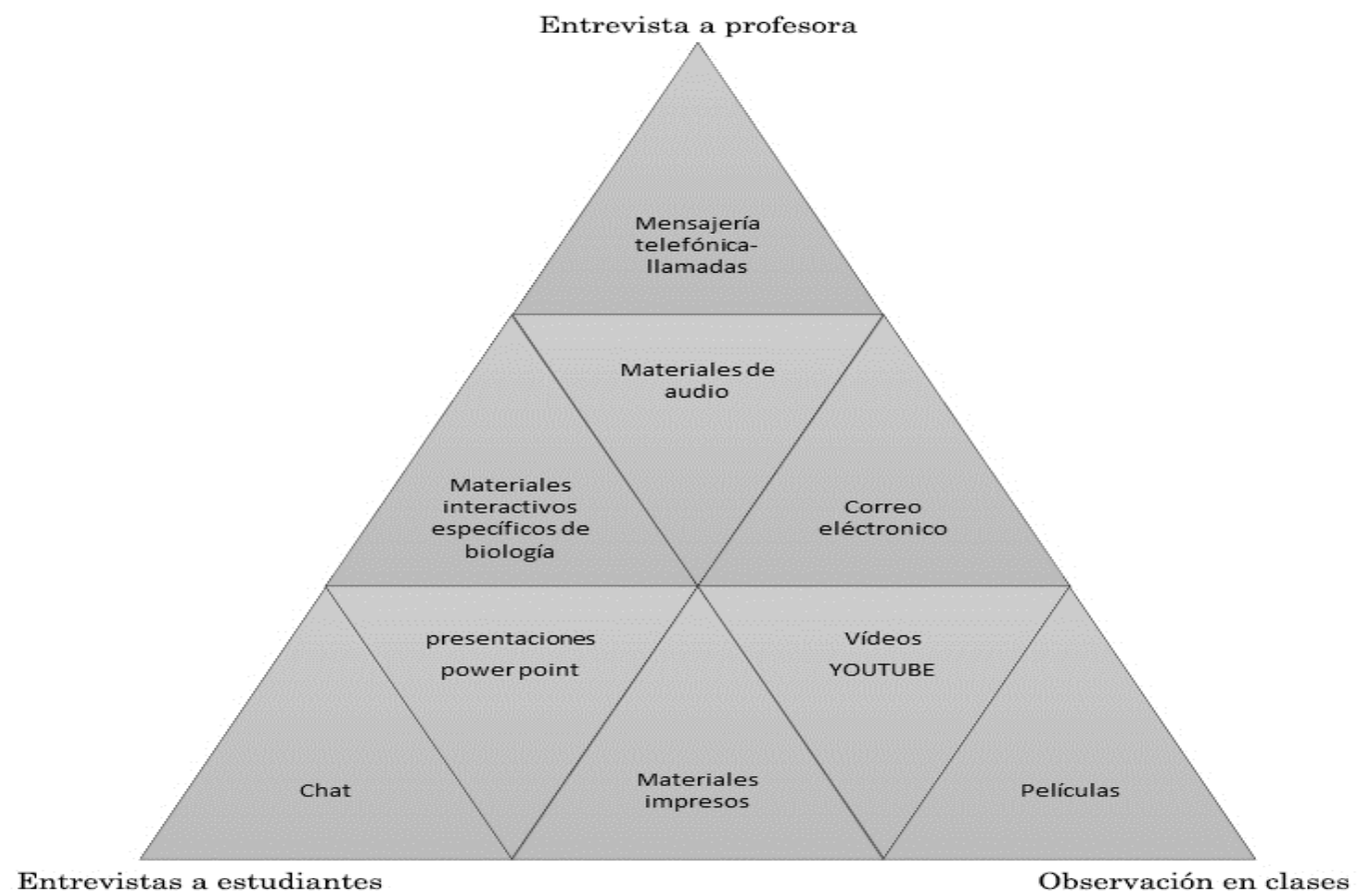

Figura 1.

Recursos usados por la docente que participó en esta investigación para la mediación pedagógica

Fuente: propia del estudio. 
Se puede observar de acuerdo a la triangulación que se señalan sobre los recursos didácticos, el uso de las TIC favorece la interacción de los estudiantes y la profesora, debido a que el centro educativo cuenta con las condiciones idóneas para poder combinar el contenido con las nuevas tecnologías, y la docente, por tener una amplia experiencia en el campo en la formación secundaria y universitaria, posee las herramientas que le permiten facilitar el aprendizaje de acuerdo con los objetivos cognitivos planteados, de tal manera, que los estudiantes se vuelven partícipes en su totalidad de los recursos disponibles, al disponer de acceso a los mismos.

Justo (2011), señala que la integración de la tecnología en la educación se ha convertido en un factor fundamental, esencial para el desarrollo cognitivo y la formación de los alumnos, lo que exige a la escuela que se adapte a las nuevas dinámicas de cambio. Por eso es importante que los profesores muestren la capacidad de flexibilidad y de comunicación con sus estudiantes, para que logren en forma conjunta, explorar los distintos medios en los que se puede acceder el conocimiento.

$\mathrm{Al}$ respecto un estudiante señala que todos los recursos usados por la profesora son muy buenos y sobre todo que ella está muy actualizada con la tecnología. Es bueno que por medio de esta herramienta podamos aprender. El correo ayuda mucho, el Messenger también, en ocasiones le llamamos, cuando algo es urgente. Realmente para mi punto de vista, las clases de Biología son muy interactivas y a la hora de explicar, la profe sabe y domina el contenido de cada tema y lo amplía de tal manera que a uno le guste, y claro, eso hace que no tengamos muchas dudas y le entendemos mejor, aunque es un poco rápido todo (EE8).

En esta misma línea, Tafur y De la Vega (2010), señalan que la dotación de infraestructura tecnológica en los colegios se está incrementando, aunque no es la solución para la mediación en el aula, porque se requiere del trabajo de capacitar a los docentes, que les permita generar conocimientos acerca de su uso, lo que demanda cambios educativos profundos que renueven paradigmas, enfoques y métodos.

Cuevas (2007), señala que en la práctica docente con los medios y materiales curriculares, han estado organizadas tradicionalmente en torno a un tipo de tecnología: material impreso, porque en los centros educativos nunca falta el libro de texto, por lo que esta supremacía está experimentando una gran transformación, provocada por los cambios introducidos por las TICs. (p.72).

Con base en lo que opinan los estudiantes sobre la revisión de artículos en revistas y la observación de vídeos o documentales, señalaron:

La verdad hay que leer muchos artículos científicos, se analizan reportajes en Discovery y se discute en clase, ejemplos de ello para decir algunos, son el origen de la vida y las especies, si el virus es un ser vivo o no, ecología y mucho más. La verdad, es una manera de entender muchas de las cosas que uno con la lectura, o con una simple lectura puede comprender, es un poco cansado, pero no se debe ver como un trabajo, sino

José Pereira-Chaves

Artículo protegido por licencia Creative Commons: BY-NC-ND / Protected by Creative Commons: BY-NC-ND

Revista de acceso y publicación gratuita/ Access and publication in Uniciencia is totally no fee. 
como algo que sirve para estudiar y aprender de una vez por todas. Es una manera linda de aprender la materia, y hasta se le vuelve hábito a uno. Aunque la profesora no diga que debemos buscar vídeos, nosotros lo hacemos para entender mejor. (EE7).

El gusto por las TIC's por parte de los estudiantes, es una oportunidad para que el docente provoque la reflexión de sus alumnos, como forma de que desarrollen habilidades necesarias que les permitan avanzar con sus propias estrategias y planificar su propio aprendizaje, como medio de promover el interés, la imaginación, la exploración, la criticidad, la reflexión, la creatividad de lo que están aprendiendo. Aquí el docente no sólo es una persona experta en el contenido que enseña, sino es promotor de cambio, es un mediador social de los estudiantes, en esta misma línea Rodríguez (1999) resalta que el profesor debe desarrollar las clases con flexibilidad organizativa, frecuentemente en grupos, y utilizar una metodología activa y participativa que propicia debates e intercambio de opiniones.

Uno de los aspectos positivos que se ha observado en la parte educativa es el aporte de la sociedad de la información, el cual ha favorecido nuevas maneras de trabajar, organizar el contenido, incentivar a los estudiantes a la exploración de nuevos conceptos y la adquisición del conocimiento. Sin embargo, en la reciente investigación de Matarrita y Serrano (2012), señalan que los centros educativos que cuentan con los recursos tecnológicos, hay muchos docentes que no tienen interés en utilizar los mismos. A la vez señalan que para los docentes estos recursos son una herramienta como cualquier otra que puede ser empleada a la hora de desarrollar las clases, pero no creen que haya beneficio alguno en el proceso de enseñanza, si se usan. Eso evidencia la apatía de muchos docentes hacia el uso de una herramienta tecnológica. Aquí cabría investigar, si es porque no tienen conocimientos suficientes sobre el uso de los equipos o si es que les genera la necesidad de emplear más tiempo ordenando, buscando y realizando las actividades para el desarrollo de los contenidos.

Sin embargo, como docente, es claro que el uso de los recursos tecnológicos favorece el plasmar y evidenciar el desarrollo conceptual en el aula, de tal manera, que se puedan potenciar las diversas formas de aprender del estudiante. De este modo se favorece la enseñanza en general, el interés y la motivación en el alumnado, elementos necesarios para lograr el aprendizaje en cada situación. Por ello Coll y Monereo (2008), señalan que la sociedad de la información permite comunicarnos, relacionarnos, aprender, pensar y hasta la forma de vivir está muy relacionada con ella. En esta misma línea es imperativo resaltar que el diseño de las técnicas didácticas van muy de la mano con los objetivos de aprendizaje y la visión que tenga el docente de la presentación del contenido, partiendo de la captación del interés del estudiante y proponiendo de una manera entretenida, mediada y sobre todo interesante para el discípulo, un contenido que muchas veces se vuelve hasta tedioso, cansado, aburrido y poco significante.

Por otra parte, Coll, Mauri y Onrubia, (2008) señalan, que cuando nos aproximamos al estudio mediante la utilización de tecnologías de la información y la comunicación, la 
planificación tecnológica facilita la mediación y comprensión del contenido de manera más atractiva para el aprendiz.

Desde esta perspectiva, se hace necesario que los profesores reflexionen, sobre cómo se da el proceso de comunicación, que permita asumir la enseñanza en su articulación con múltiples formas de expresión, desde el lenguaje propio, el lenguaje de los otros, la informática, los medios de comunicación de masas, en fin, la comunicación en su totalidad.

No obstante, en términos generales se habla de que el uso de la tecnología potencia la alfabetización, pero se debe tener cuidado con ello, sobre todo pensando en la resistencia que pueden tener algunos docentes frente al uso adecuado de la tecnología, como recurso de enseñanza y aprendizaje. Un estudio reciente, publicado en el British Educational Research Journal, analizó datos de más de 47000 participantes recogidos en las encuestas anuales del National Institute of Adult and Continuing Education (NIACE), donde el Dr. White (2012), señala que el análisis aporta evidencia que sugiere que las barreras que impiden la participación en actividades de aprendizaje son actitudinales, y no prácticas, logísticas o económicas (p. 167).

El aprendizaje en la adultez parece estar asociado a las actitudes positivas hacia la educación formada durante el período de escolarización obligatoria. Esto quiere decir que aquellos jóvenes que experimentaron fracasos educativos o fueron alienados por el sistema escolar, difícilmente se deciden a participar en actividades formativas como adultos, independientemente de las oportunidades o los beneficios potenciales. Esto es muy importante, porque este estudio brinda evidencia de que se debe usar y maximizar el uso de la tecnología con los estudiantes, como una forma de incentivarles la formación continua y permanente en su actividad profesional.

Considerando las estrategias metodológicas y técnicas didácticas que usaba la profesora durante el desarrollo de la clase, en una de las entrevista una estudiante señaló:

Todo el material que se utiliza en clases por parte de la profesora es excelente, me encanta porque es muy diverso. Por ejemplo la semana pasada se vio vídeo, la antepasada se usó proyector, nos llevó al laboratorio, se hacen mapas conceptuales o esquemas etc.; me gusta mucho; no me aburre como la profesora da clases, que se me van volando y eso es bueno, porque significa que se ha aprovechado. (EE12)

Por otra parte en el trabajo del grupo focal (GF) se resalta algunos puntos:

La profesora acude a diversas técnicas didácticas que ayudan mucho a la introducción del tema, aunque tenemos un libro de Biología de texto universitario, no se conforma con ese. Nos mandaba a utilizar todos los libros de Biología que usted sabe que hay. Realmente nos ubicaba en el capítulo del libro de texto y nosotros nos encargábamos de buscar otros libros y revisarlos, eso nos enseña a poder ir ambientándonos en la vida universitaria. (GF). 
Uniciencia. Vol. 29, No. 2, pp. 62-83. Julio-Diciembre, 2015. URL: www.revistas.una.ac.cr/uniciencia

Email: revistauniciencia@una.cr
ISSN Electrónico: 2215-3470

DOI: http://dx.doi.org/10.15359/ru.29-2.5

En enseñanza de la Biología, el docente articular permanentemente los contenidos procedimentales, actitudinales y conceptuales. Éste debe atender la educación integral del estudiante, de manera que se formen ciudadanos con capacidad de análisis, alto poder de relacionar datos, conceptos y desarrollar habilidades que les permita desenvolverse socialmente de manera activa y competente de forma reflexiva y crítica. Esta preocupación de integrar los contenidos se viene trabajando desde 1992, por ejemplo con Coll et al (1992) quienes sugieren la interrelación de los tres tipos de contenidos, lo cual ha tenido un auge importante de reflexión dentro de la comunidad educativa.

La coherencia del accionar pedagógico con el compromiso social que se adquiere en profesión docente, debe estar claramente determinada en cada uno de nosotros, partiendo de la concepción de la alfabetización científica. Debemos procurar que la población cuente con los conocimientos de los contenidos científicos mínimos, para que como ciudadanos puedan actuar democrática y responsablemente.

El docente con formación científica debe adaptar los objetivos conceptuales, para que los alumnos aprendan ciencias, de manera que se incentiven los procesos paralelos de aprender y hacer, por lo son importantes las actividades que se desarrollan. En la figura 2 se hace referencia al desarrollo de las clases de Biología:

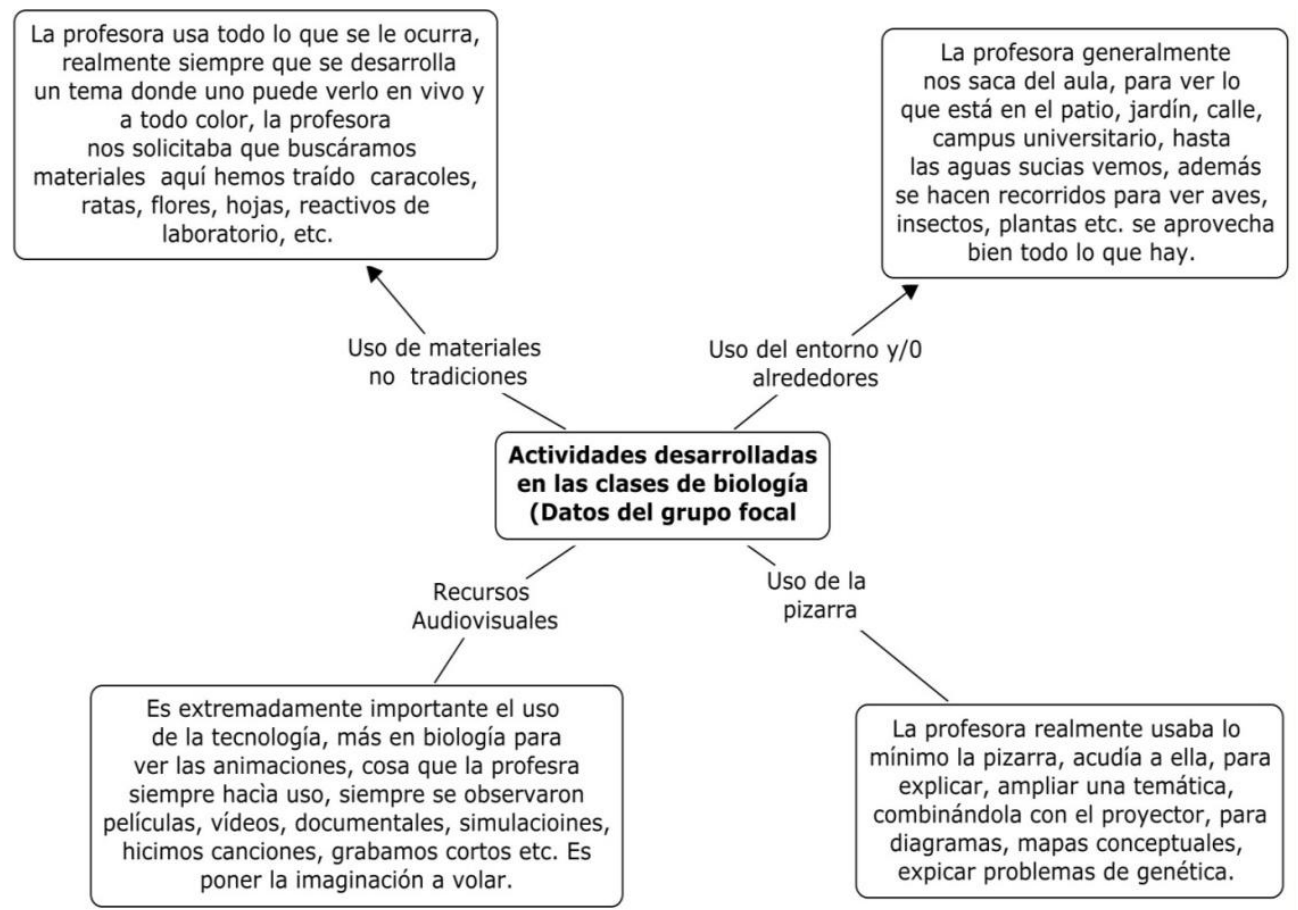

Figura 2.

Actividades desarrolladas en las clases de Biología por la docente que participó en esta investigación.

Fuente: propia del estudio. 
Por otra parte se resalta que todo el acto educativo registrado durante la investigación etnográfica, conlleva la sinergia de las distintas metodología y recursos de aprendizaje, por lo que los materiales de textos, guías de trabajo, manuales, el aprovechamiento de todos los elementos para desarrollar el contenido, responden a la complementariedad del uso de la tecnología para su presentación, desde el diseño gráfico hasta la utilización de material de audio o vídeo-conferencias; recursos a los que se tiene acceso y el aprovechamiento por los estudiantes y el docente hacen que realmente se logre potenciar la tecnología, como un recurso que favorece el aprendizaje.

Se destaca la variedad y diversidad de programas visuales y materiales o paquetes computacionales, que facilitan el logro de la comprensión e interiorización de los contenidos. Esto se alcanza en gran parte, con los aportes del profesor o la colaboración institucional del personal, entre la profesora del curso, el profesor de informática y los estudiantes, quienes hacen converger los fines del aprendizaje, para que se hagan una realidad.

Los educadores enfrentan constantes innovaciones, que son importantes para el desarrollo de su práctica docente, y así la integración de las tecnologías como un recurso en el proceso de enseñanza y aprendizaje, siempre debe ser un elemento académico que favorece una actitud positiva y creativa en los alumnos, operando en ellos una verdadera transformación en el proceso de enseñanza y aprendizaje, al promover el papel protagónico.

Los recursos tecnológicos, son de gran atracción para los adolescentes, razón por demás interesante para tratar de plantear estrategias que los utilicen para influir positivamente en los procesos de enseñanza y aprendizaje de los estudiantes y en los procesos de planeamiento y desarrollo de clases de los docentes.

Así mismo, entre los datos más sobresalientes de las entrevistas realizadas a estudiantes se evidenció la necesidad de que las clases sean más vivenciales, experimentales e indagatorias, aspecto que se puso de manifiesto con la pregunta: ¿Para usted las prácticas de laboratorio son importantes para el desarrollo de las clases de Biología? Entre algunas de las respuestas se tienen manifestaciones sobre la necesidad de trabajos prácticos (figura 3): 
Uniciencia. Vol. 29, No. 2, pp. 62-83. Julio-Diciembre, 2015. URL: www.revistas.una.ac.cr/uniciencia Email: revistauniciencia@una.cr
ISSN Electrónico: 2215-3470 DOI: $\underline{\text { http://dx.doi.org/10.15359/ru.29-2.5 }}$

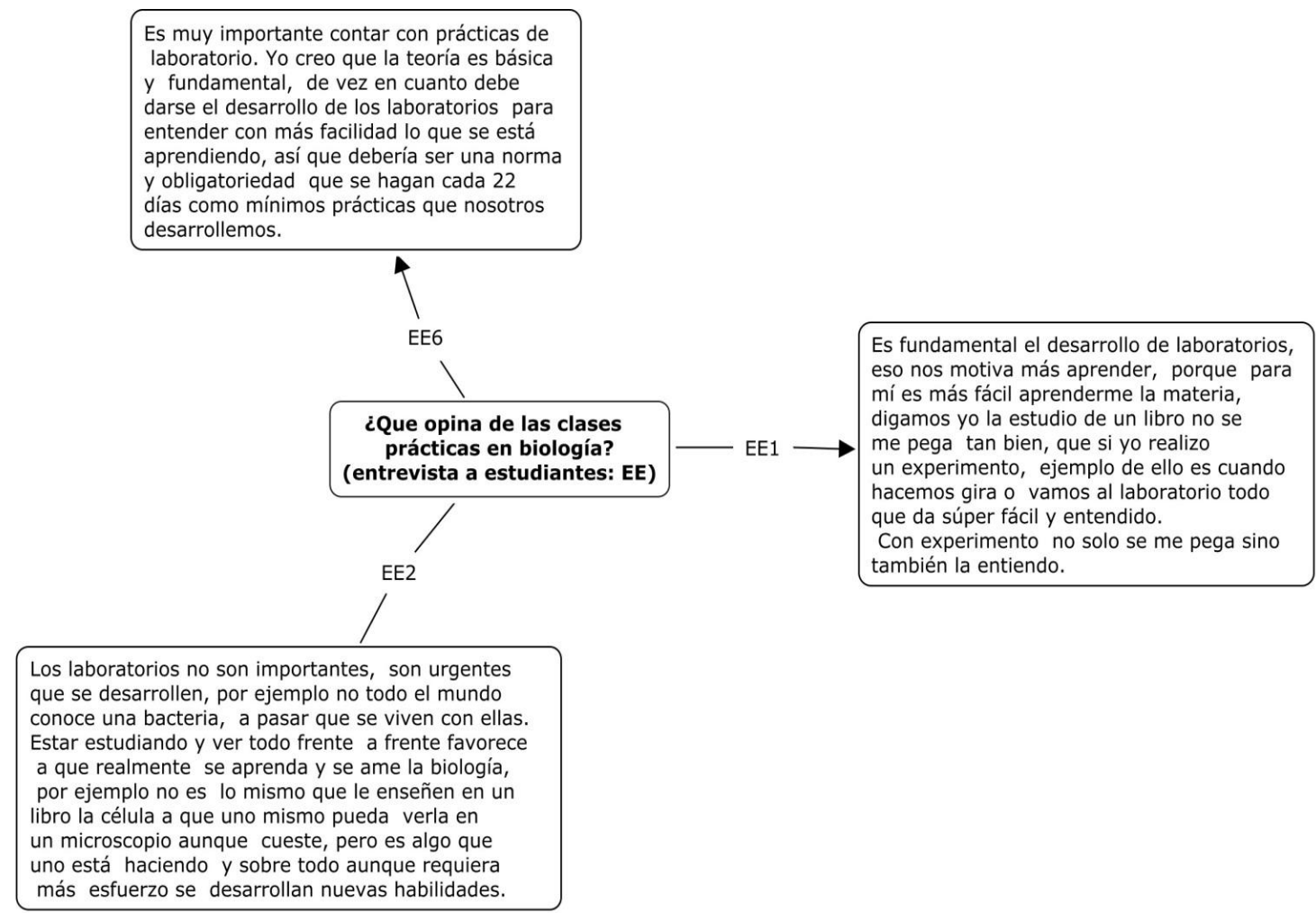

Figura 3.

Opinión de los estudiantes sobre las clases prácticas de Biología. Fuente: propia del estudio.

Por ejemplo, con base en las expresiones anteriores, en la figura 4 se evidencia el asombro de los estudiantes con la interacción de los distintos especímenes durante un laboratorio:

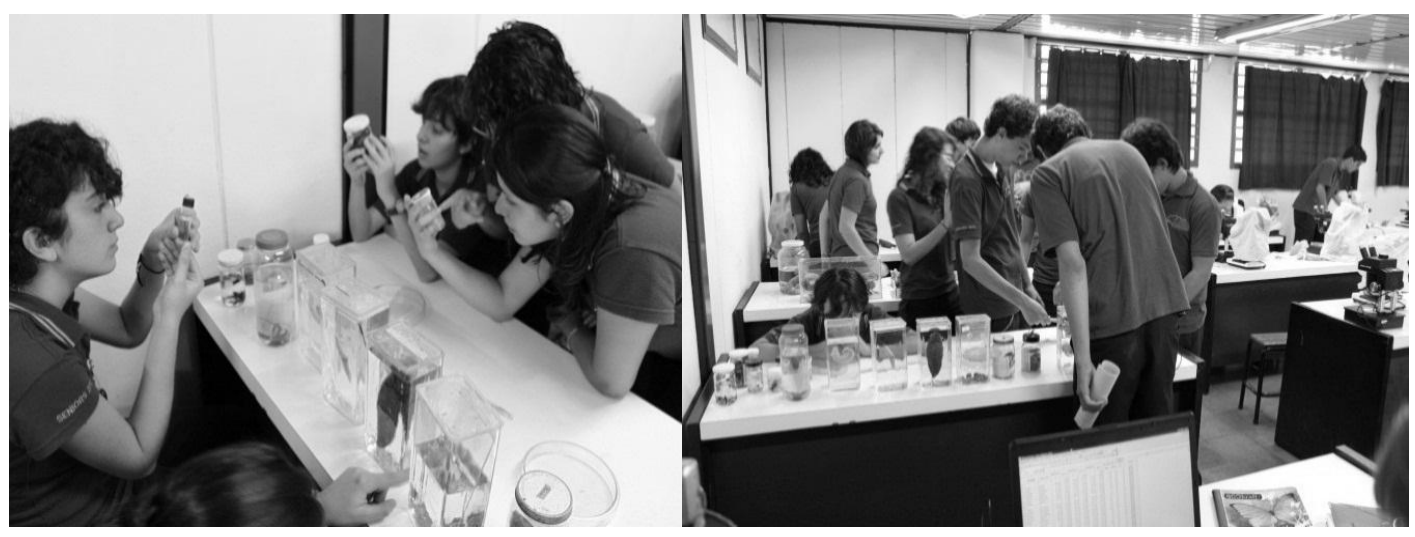

Figura 4.

Desarrollo de una práctica de laboratorio en la Escuela de Ciencias Biológicas-UNA Fuente: propia del estudio.

José Pereira-Chaves

Artículo protegido por licencia Creative Commons: BY-NC-ND / Protected by Creative Commons: BY-NC-ND

Revista de acceso y publicación gratuita/ Access and publication in Uniciencia is totally no fee. 
Las estrategias metodológicas en donde se aplican los trabajos prácticos favorecen la formación del estudiantado, sobre todo pensando que el docente de disciplinas científicas debe promover el trabajo científico, para que el estudiante se identifique con lo que está desarrollando, de manera que sea capaz de aplicar los principios básicos del método científico, que utilice adecuadamente el material, los instrumentos y que aplique técnica didáctica básicas de laboratorio. Y a la vez, que con este tipo de trabajo, se fortalezca la actividad en equipo y se potencien las destrezas y habilidades de interpretación de datos. De esta manera, los estudiantes adquieren habilidades en escritura, sistematización de datos y muchas veces adoptan un papel protagónico o de liderazgo con los trabajos prácticos figura 5, razón por la cual se busca en los laboratorios promover objetivos:

\begin{tabular}{|c|c|c|c|c|c|}
\hline 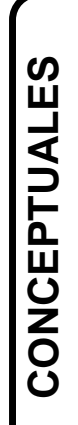 & $\begin{array}{l}\text { Aplicación de aspectos } \\
\text { teóricos desarrollados } \\
\text { en el aula. } \\
\text { Reformulación } \\
\text { conceptual de términos } \\
\text { teóricos. } \\
\text { Contrastación de } \\
\text { hipótesis. } \\
\text { Cuerpo conceptual de } \\
\text { conocimiento. }\end{array}$ & $\infty$ & $\begin{array}{l}\text { Desarrollo de } \\
\text { habilidades prácticas. } \\
\text { Se promueve la } \\
\text { investigación y el } \\
\text { análisis. } \\
\text { Se desarrollan } \\
\text { habilidades de } \\
\text { comunición. } \\
\text { Metodología de la } \\
\text { investigación. }\end{array}$ & & $\begin{array}{l}\text { Se genera un espíritu } \\
\text { de colaboración y de } \\
\text { trabajo en equipo. } \\
\text { Se propicia el interés } \\
\text { hacia las ciencias } \\
\text { biológicas. } \\
\text { Se promueva mayor } \\
\text { interés hacia la } \\
\text { apliación conceptual. } \\
\text { Actitud científica. }\end{array}$ \\
\hline
\end{tabular}

Figura 5.

Dimensiones consideradas en el desarrollo del contenido de Biología en el salón de clases. Fuente: propia del estudio.

Es notorio que el abordaje de los contenidos de una manera repetitiva no favorece la motivación en los estudiantes. Por ello, el desarrollo de prácticas de laboratorio o giras de campo promueven la inquietud de indagar más sobre la temática, de modo que la conceptualización es medular para la bioalfabetización, en la cual, los temas de equilibrio humano, evolución y biodiversidad, homeostasis de la naturaleza y construyamos el futuro fueron abordajes teóricos que los estudiantes experimentaron como vivencia en las giras y en los laboratorios.

Para lo fomentar el contenido procedimental aplicaron la observación, por medio de la cual clasificaron individuos, formularon una serie de preguntas, registraron sus datos para la elaboración de un informe, mediante el cual comunicaron los resultados, la rigurosidad, destrezas, habilidades organizativas, que grupalmente fueron fundamentales para el logro de estos objetivos procedimentales. Con base en la parte actitudinal, una de las cosas que se resaltaron es el entusiasmo y respeto con que los estudiantes desarrollaron

José Pereira-Chaves

Artículo protegido por licencia Creative Commons: BY-NC-ND / Protected by Creative Commons: BY-NC-ND

Revista de acceso y publicación gratuita/ Access and publication in Uniciencia is totally no fee. 
cada una de las actividades dentro y fuera del aula, hay un marcado respeto hacia la profesora, y hacia cada uno de los compañeros; los estudiantes tienen visiones distintas y las comparten, discuten reflexivamente, hay una visión de respeto, compromiso y mucha sensibilidad hacia la naturaleza y la vida silvestre en general, aspecto observado en el laboratorio y también en las giras.

La aplicación de los contenidos procedimentales favorecen a que se generen actitudes positivas hace el aprender la biología, elemento que promueve la profesora en sus estudiantes; los abordajes conceptuales, metodológicos y actitudinales se observa en la figura 6.
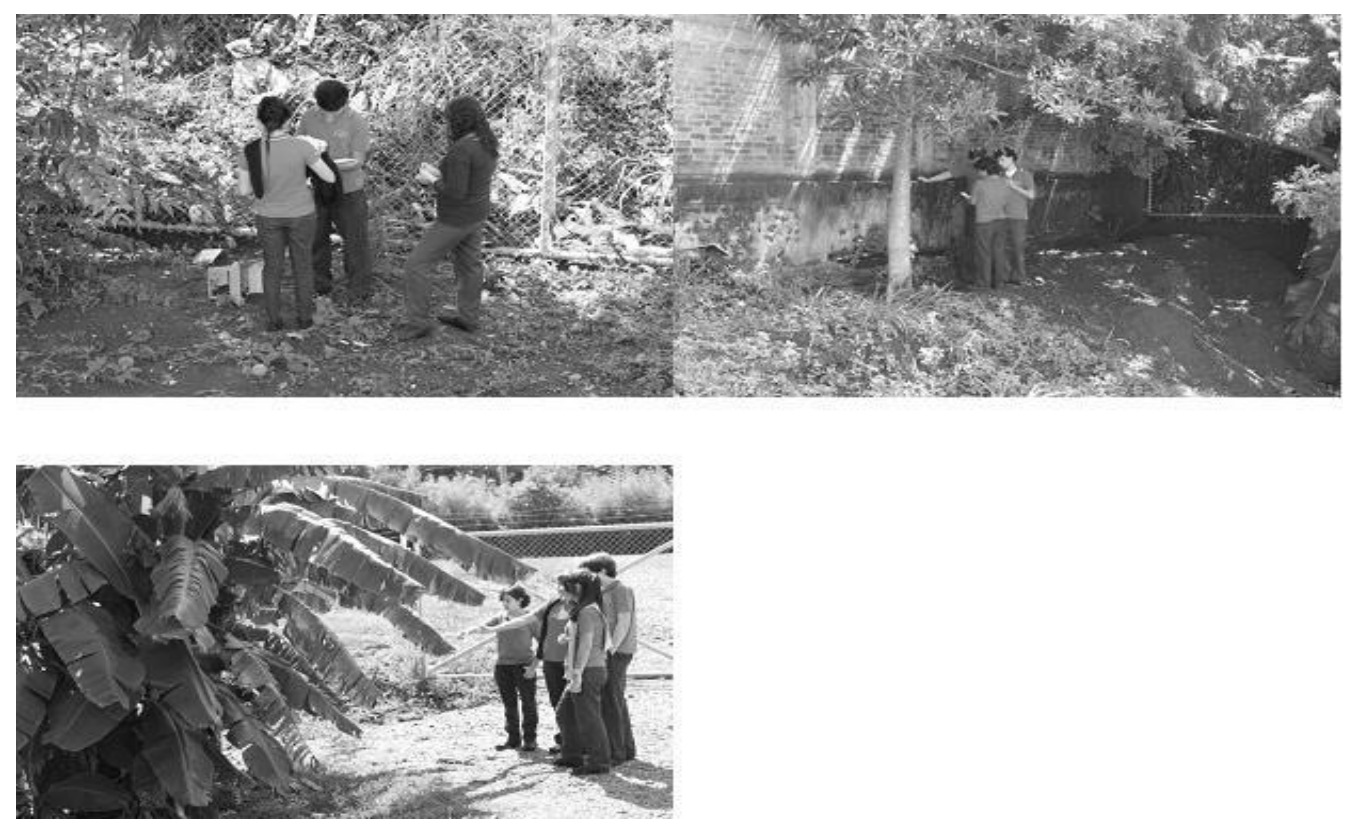

Figura 6.

Trabajos de los estudiantes fuera del aula, aplicando los conceptos desarrollados en clases.

Fuente: propia del estudio.

Con la ilustración de la figura anterior y en las observación realizada en la actividad de aprendizaje se evidencia la sistematización, comunicación y el trabajo en equipo que se lleva a cabo en el desarrollo de los conceptos, lo que responde a los intereses de los estudiantes; con la aplicación de la teoría y la práctica se da la articulación del desarrollo de los contenidos curriculares de Biología y a la vez se generan o ponen en práctica actitudes de valoración de la naturaleza y la conservación, además, una de las formas de vivenciar los contenidos en Biología es a través de las giras educativas, lo cual son muy importantes como técnicas didácticas para el desarrollo conceptual, actitudinal y metodológico(figura 7). 


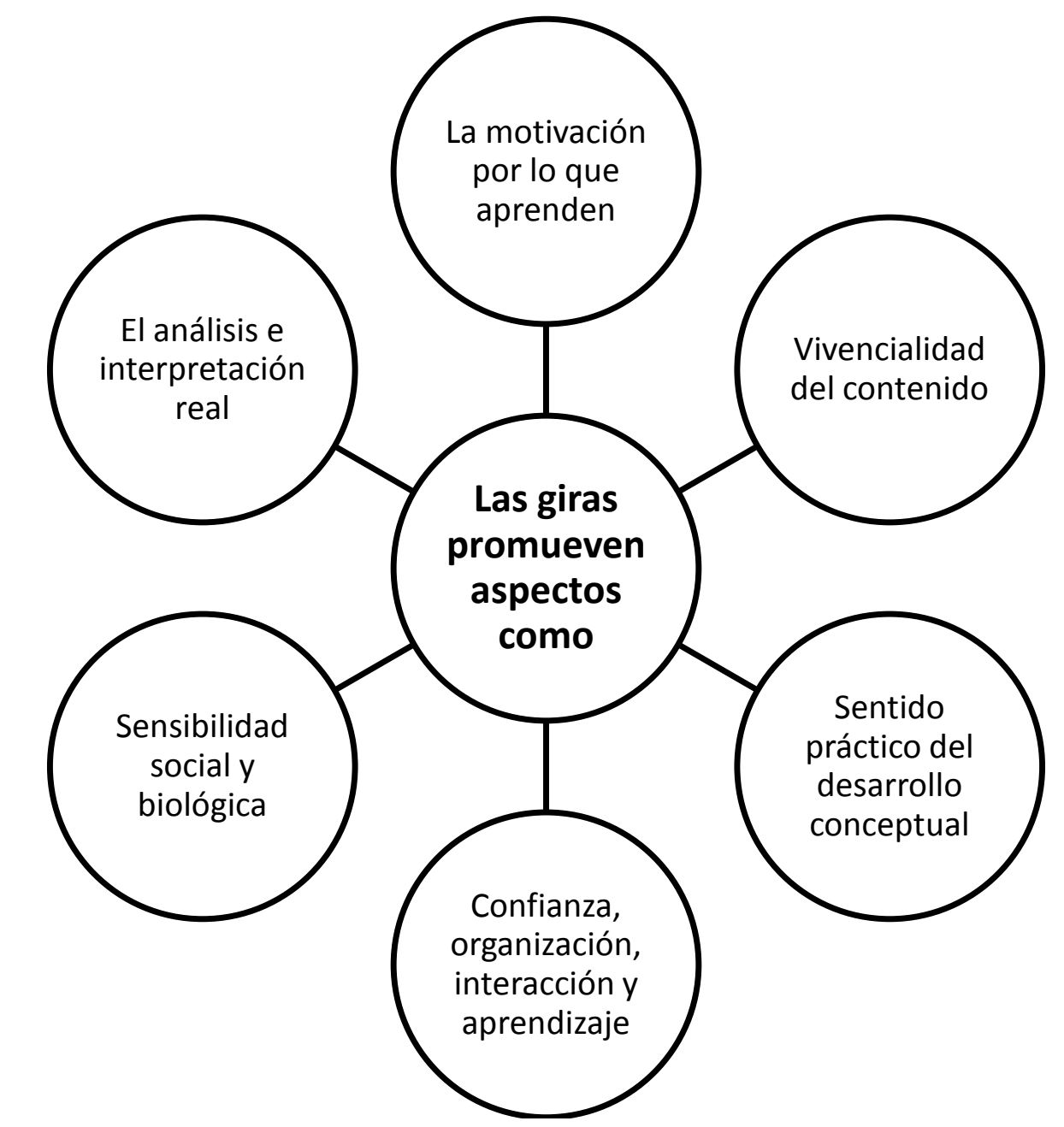

Figura 7.

Aspectos que promueven las giras, según los estudiantes Fuente: propia del estudio.

En las giras, la docente busca utilizar problemas aplicados a las ciencias biológicas, para que los estudiantes apliquen lo aprendido y propongan alguna aplicación práctica. Ellos tienen que mostrar un grado de integración de los contenidos abordados y el grado de aprendizaje relevante que se ha producido durante el desarrollo en el aula y fuera de ella. Así, las giras educativas o trabajos de campo contribuyen a la formación de ciudadanos que puedan cuidar de sí mismos y de los demás, además Pereira, Camacho, Muñoz (2013) señalaron que los trabajos en el campo favorecen la aplicación de los principios teóricos, técnicos y científicos que se busca promover en los estudiantes y, sobre todo, permiten sembrar la semilla para que se interesen por las ciencias de la vida o afines a esta, teniendo en cuenta la necesidad de potenciar los jóvenes científicos en el país y que se gesten, a corto plazo, trabajadores científicos con una capacidad crítica y analítica. 
Uniciencia. Vol. 29, No. 2, pp. 62-83. Julio-Diciembre, 2015.

Con base en la opinión de los estudiantes y el grupo focal sobre la relación entre la teoría y la práctica desarrollada por la profesora durante las clases, se obtuvo como datos comunes entre las entrevistas, y el grupo focal lo siguiente:

Ellos señalaron que la teoría debe ser vivenciada con prácticas de laboratorio y de campo, sino es muy aburrido, lo que si destacan que su profesora fue muy cuidadosa en ese sentido, y que eso se refleja con el hecho de que la profesora tenga tanto conocimiento y relacione lo que desarrolla con lo que ellos saben es muy positivo, y sobre todo que les ayuda a comprender la materia y a la vez les motiva saber que estaban familiarizados con lo que se estaba desarrollando.

Otro de los hallazgos obtenidos de las experiencias de campo que tuvieron los estudiantes es la siguiente:

Consideran que una de las cosas más interesantes del colegio son las giras que se realizan, las cuales son apoyadas con el transporte de la Universidad. Consideraron que tales actividades son muy interesantes, debido a que aplicaban todo lo aprendido en las clases, a la vez que desarrollaban trabajos aplicados a las diversas formas de vida. También se refirieron a que todos los alumnos en el país deberían tener la oportunidad de participar en giras educativas, ya que se salen del esquema de enseñanza efectuada solamente en el aula. (GF)

Basado en las entrevistas realizadas a los estudiantes con la pregunta de ¿Cómo consideran las estrategias de enseñanza que utiliza la profesora, para que logren aprender y ampliar sus conocimientos en Biología?, se obtuvo lo siguiente (figura 8). 
Uniciencia. Vol. 29, No. 2, pp. 62-83. Julio-Diciembre, 2015. URL: www.revistas.una.ac.cr/uniciencia

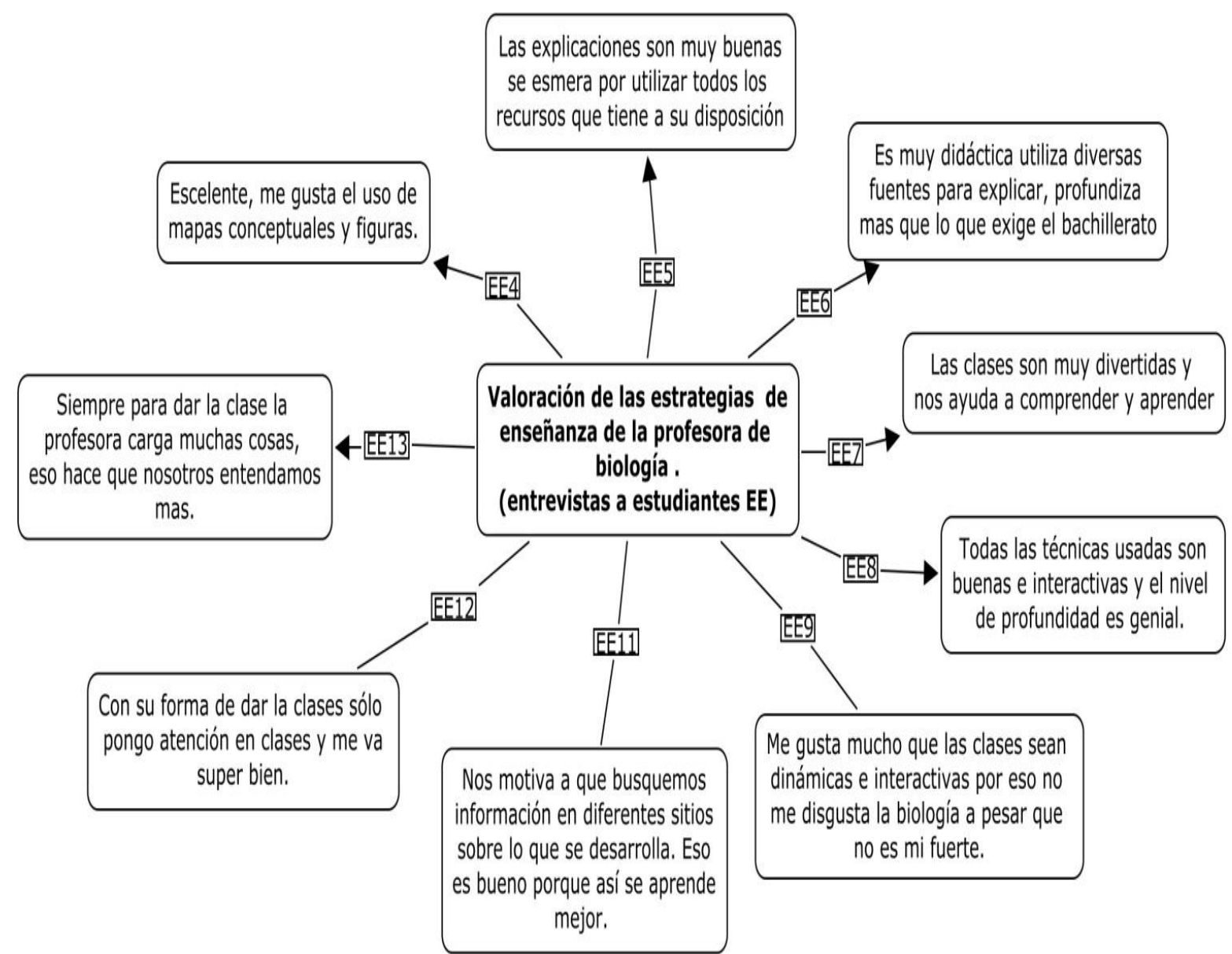

Figura 8.

Valoración de las estrategias de enseñanza, utilizadas por la docente en el salón de clases de Biología.

Fuente: propia del estudio.

Por otra parte, la expresión de una estudiante señaló:

Antes era un poco más difícil para un profesor dar clases, porque no había internet, ni otros instrumentos didácticos con los cuales contamos actualmente. Estábamos acostumbrada a un libro y guías de trabajo, pero aquí logré cambiar totalmente el esquema de aprender Biología; por eso considero que fue muy buena idea la de decidir matricularme en este colegio, y me siento muy satisfecha de poder tener a disposición una gran cantidad de recursos didácticos (EE10).

La profesora señala que sus clases y las demás lecciones deben ser basadas en los principios humanísticos, que motivan a los jóvenes con vocación hacia el pensamiento creativo, las artes y las letras, porque se debe propiciar un modelo educativo centrado en la 
persona, en sus cualidades y sus experiencias y así tener jóvenes bioalfabetizados. Tal modelo estimula al estudiante a conocerse y a decidir lo que quiere llegar a ser. (EP3)

Al respecto sobresalen los siguientes estudios, entre los cuales, Oxford (2002), establece que el uso de diferentes estrategias es considerado como uno de los principales factores que permiten un aprendizaje exitoso, y esto es una forma de aumentar el conocimiento científico en los estudiantes, promoviendo la bioalfabetización. Por su parte Ramos (2004) señala que la bioalfabetización hace referencia a los conocimientos, habilidades y saberes presentes en el mundo de las ciencias y la tecnología. Vieira (2007) señala que ahora se tiene por objetivo educar a los ciudadanos informados, capaces de participar en debates científicos, atentos a las causas y consecuencias inherentes al conocimiento y su aplicación en la vida cotidiana.

Para los centros educativos y los profesores es primordial promover una nueva forma de gestar el conocimiento, mediante la creación de ambientes de aprendizaje que estimulen el desarrollo y el interés de aprender; donde las tecnologías y los recursos didácticos complementarios contribuyan de manera integral hacia el aprender haciendo y aprender aprendiendo.

\section{Conclusiones}

Se evidenció en las diversas técnicas de recolección de datos, que tanto la profesora como los estudiantes consideraban que el temario de Biología de Educación Diversificada $\left(10^{\circ}, 11^{\circ}\right.$ y $12^{\circ}$ año $)$ es muy amplio y difícil de abarcar en su totalidad, por lo que se deben priorizar los contenidos de acuerdo con la complejidad y secuencia de su abordaje. Tal aspecto fue considerado por la profesora en el desarrollo de las diversas actividades desarrollados como trabajos extra clases y giras de campo, para aplicar tanto el contenido priorizado en el aula, como el que no ha sido desarrollado, por el poco tiempo con que cuenta semanalmente, lo que ha promovido el interés de los estudiantes por la materia.

La docente pone en práctica los conocimientos previos de sus estudiantes, rescatando las vivencias de cada uno y la articulación de aspectos propios de la dinámica social. La habilidad de la facilitadora se centró en conocer el contexto en que se desarrolla el contenido, considerando que hay diferentes maneras de iniciar la construcción de los nuevos conocimientos que se van sistematizando de forma progresiva y sobre todo, estimulando la socialización de los contenidos en el aula.

La planificación didáctica como herramienta de trabajo, le permitió a la docente apropiarse de los diseños curriculares, contextualizando las disposiciones formuladas para todos los alumnos del centro educativo, además de identificarse con la filosofía de la institución, donde siempre promovía en sus estudiantes la parte conceptual y procedimental, despertando en ellos actitudes positivas hacia el campo biológico, todo lo cual se reflejó en los métodos y técnicas de trabajo en el aula. 
Uniciencia. Vol. 29, No. 2, pp. 62-83. Julio-Diciembre, 2015.

\section{Referencias}

Acosta, S. y Acosta, R. (Junio, 2010). Los mapas conceptuales y su efecto en el aprendizaje de conocimiento biológico. Revista Omnia. 16(2), 209-225. Recuperado de http://www.redalyc.org/pdf/737/73715084012.pdf

Allen, J. (2006). Emotional Intelligence in Classrooms and in Schools: What We See in the Educational Setting. En K. R. Murphy, A critique of Intelligence: What are the problems and how can they be fixed? (Series in Applied Psychology)(pp. 125-139). Mahwah, New Jersey: Lawrence Erlbaum Associates. Inc.

Alterio, G. y Ruiz, C. (2010). Mediación metacognitiva, estrategias de enseñanza y procesos de pensamiento del docente de Medicina. Revista Educación Médica Superior, 24(1), 25-32. Recuperado de http://scielo.sld.cu/pdf/ems/v24n1/ems04110.pdf

Coll, C y Monereo, C. (2008). Educación y aprendizaje en el siglo XXI: Nuevas herramientas, nuevos escenarios, nuevas finalidades. En C. Coll y C. Monereo (Eds.), Psicología de la educación virtual: Aprender y enseñar con las tecnologías de la información y la comunicación, 2, 20-51. Madrid, España: Morata.

Coll, C., Mauri, T. y Onrubia, J. (2008). La utilización de las tecnologías de la información y la comunicación en la comunicación en la educación. Del diseño tecno-pedagógico a las prácticas de uso. En C. Coll y C. Monereo (Ed.), Psicología de la educación virtual: Aprender y enseñar con las tecnologías de la información y la comunicación, 3, 75-103. Madrid, España: Morata.

Coll, C., Pozo, J. I., Sarabia, B. y Valls, E. (1992). Los contenidos en la Reforma. Enseñanza y aprendizaje de conceptos, procedimientos y actitudes. Ed. Santillana. Madrid, pp. 81-132

Cuevas, A. (2007). Lectura, alfabetización en información y biblioteca escolar. Gijón. Trea.

Dale, S y Ueckert, C. (2007). Teaching biology.The great dilemma. Journal of biological Education, 41(2), 51-52. DOI http://dx.doi.org/10.1080/00219266.2007.9656061

Díaz, J. (2002). El Método Comparativo en la Biología Evolutiva. Etología, Madrid, Revista Etologuía, $19 \quad$ (20), 1-46. $\quad$ Recuperado de http://www.ucm.es/data/cont/media/www/pag-33471/2001_Etologuia_19_37.pdf

Justo, J. (2011). Contribuição de um blog na promoção da literacia científica no ensino da física e da química no 3 . $^{\circ}$ ciclo do ensino básico. Tese de mestrado, Educação (Didáctica das Ciências), Universidade de Lisboa, Instituto de Educação. 
Uniciencia. Vol. 29, No. 2, pp. 62-83. Julio-Diciembre, 2015.

Martínez, M., García, B. y Quintana, J. (2006). El perfil del profesor universitario de calidad desde la perspectiva del alumnado. Revista Educación XXI. 9(1), 183-198.

Matarrita, L. y Serrano, J. (2012). Estrategias didácticas Utilizando recursos tecnológicos en la implementación del eje transversal cultura ambiental para el desarrollo sostenible en un grupo de sétimo año del tercer ciclo de la educación básica. (Tesis de licenciatura en Enseñanza de las Ciencias). Universidad Nacional de Costa Rica. P.120.

Oxford, R. (2002). Metodología en la enseñanza: una antología de la práctica actual de Nueva York. EEUU: Cambridge.

Pereira, J. (2010). La enseñanza de Biología en Costa Rica: Los Retos del abordaje pedagógico para la alfabetización científica y la motivación estudiantil en Secundaria. Congreso Iberoamericano de Educación. Buenos Aires, Argentina.

Pereira, J., Camacho, S. y Muñoz, N. (2013). La Olimpiada Costarricense de Ciencias Biológicas y su papel en la comunidad educativa nacional mediante la integración del competir, compartir, convivir y aprender. Revista Uniciencia. 27 (1), 245-265

Ramos, M. (2004). Informe Projecto Gulbenkian. Formação de Professores. A literacia científica: Uma necessidade urgente; Um desafio a Escola. Um contributo para o painel-Aprendizagens curriculares de painel, Literacias e Biblioteca Escolares no Projeto Gulbenkian: Formação de Professores Responsáveis pelo Desenvolvimento de Bibliotecas escolares. Portugal. Recuperado de http://www.theka.org/docs/publicacoes/literacia_cientifica.pdf

Rodríguez, G., Gil, J. y García, E. (1996). Metodología de la Investigación Cualitativa. Málaga: Aljibe.

Rodríguez, J. (1999). El diseño y el desarrollo curricular en la educación secundaria obligatoria. Revista educación XX1, (2), 1-25. Recuperado de http://espacio.uned.es/revistasuned/index.php/educacionXX1/article/view/371/323

Tafur, R. y De La Vega, A. (2010). El acceso a los recursos educativos por los docentes de educación secundaria: un estudio exploratorio. Educación, 20(37), 29-46.

Recuperado de http://revistas.pucp.edu.pe/index.php/educacion/article/view/2550/2494

Valbuena, E. (2008). El conocimiento didáctico del contenido biológico. Estudio de las Concepciones disciplinares y didácticas de futuros docentes de la universidad pedagógica nacional (Colombia). Revista de Educación en Biología. 11(2), 60-63.

Vieira, N. (2007). Literacia Científica e Educação, de Ciência. Revista Lusófona de Educação, 10(1), 97-108. Recuperado de http://revistas.ulusofona.pt/index.php/rleducacao/article/view/636/531 
Uniciencia. Vol. 29, No. 2, pp. 62-83. Julio-Diciembre, 2015.

URL: $\underline{\text { www.revistas.una.ac.cr/uniciencia }}$

ISSN Electrónico: 2215-3470

Email: revistauniciencia@una.cr

White, P. (2012). Modellig the learning divide: Predicting participation in adult learning and future learning intentions 2002 t0 2010. Rev. Britsh Educational Research Journal. $\quad 1 \quad$ (38), $153-175 . \quad$ Recuperado de http://www.tandfonline.com/doi/full/10.1080/01411926.2010.529871\#tabModule

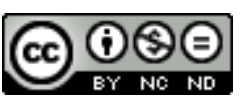

Las estrategias metodológicas en el aprendizaje de la biología (José Pereira-Chaves) por Revista Uniciencia se encuentra bajo una Licencia CreativeCommons Atribución-NoComercial-SinDerivadas 3.0 Unported 